\title{
Infrared chemical mapping of degradation products in cross-sections from paintings and painted objects
}

\author{
Eric J. Henderson ${ }^{1 *}$, Kate Helwig ${ }^{1}$, Stuart Read ${ }^{2}$ and Scott M. Rosendahl ${ }^{2}$
}

\begin{abstract}
Paintings and painted objects are quite susceptible to degradation, as paint layers are usually composed of complex mixtures of materials that can participate in chemical degradation processes. The identification of the constituent materials in paint (including binders, pigments, and fillers) and the degradation products within paint layers is of particular importance to ensuring the conservation of paintings, by providing important information both about their material history as well as their state of conservation. Metal fatty acid salts (metal soaps) are degradation products that can form in situ from interactions between inorganic pigments and free fatty acids in oil-based binding media, and can cause significant condition issues in paintings. Fourier transform infrared (FTIR) spectroscopy is one of the leading analytical techniques for the study of metal soaps. In this article, the materials analysis of several cross-sections from paintings and painted objects from works in Canadian collections is presented. Recent results on the use of external reflection FTIR (R-FTIR) spectroscopy to identify and map the distribution of paint components and metal soap degradation products is presented. In particular, zinc, lead, calcium, and copper fatty acid salts were all readily identified in paint cross-sections by R-FTIR spectroscopy, along with several pigments and the oil binding medium. The results shown here are among the first detailed examinations of these metal soaps in paint cross-sections using R-FTIR spectroscopy. The use of highly polished samples in which specular reflection is dominant allowed for spectral transformations to be applied to generate transmission/absorption-like spectra which facilitated identification of these species. The distribution of these species across the cross-sections was mapped by integrating characteristic absorption features in the R-FTIR spectra. Attenuated total internal reflection (ATR) FTIR spectroscopy was also performed on several samples, which provided additional compositional details at the interface of paint layers and degradation products.
\end{abstract}

Keywords: Metal soaps, Paintings, Degradation products, FTIR mapping, External reflection FTIR spectroscopy, Zinc soaps, Lead soaps, Calcium soaps, Copper soaps

\section{Introduction}

Works of art and cultural heritage objects are continuously subjected to degradation processes that can significantly compromise their integrity, function and aesthetic quality. Documenting the composition of these objects through detailed chemical and materials analysis is one of the first steps in ensuring their conservation, by providing important information both about their material

\footnotetext{
*Correspondence: eric.henderson@canada.ca

${ }^{1}$ Canadian Conservation Institute, 1030 Innes Rd., Ottawa, ON K1B 4S7, Canada

Full list of author information is available at the end of the article
}

history as well as their state of conservation. In the case of paintings and painted objects, paint layers are usually composed of complex mixtures of materials that can participate in chemical degradation processes. The identification of the constituent materials in paint (including binders, pigments, and fillers) and the degradation products within paint layers is of particular importance, as it can further our understanding of the chemical species, interactions, and mechanisms that are involved in these processes, inform treatment plans to address and target specific problems, advise on display and storage conditions, and advance the knowledge of the materials and techniques of the artists who created the works. 
In the past several decades, the study of metal carboxylate salts (metal soaps) in paintings has garnered tremendous attention in the art and conservation communities [1-3]. Although metal soaps are sometimes added to paints as extenders or processing aids (e.g. aluminum stearate) [4], they can also form in situ from interactions between inorganic pigments and free fatty acids in oilbased binding media. Of particular concern, they have been shown to be mobile within the paint layers, and can lead to flaking, interlayer adhesion failure, and phenomena such as protrusions and surface haze. The resulting structural and visual changes associated with their formation and accumulation is of significant concern for the long-term stability and appearance of the objects, and a focal point for interventive conservation treatment.

Lead and zinc-based soaps have been extensively studied $[5,6]$, mostly owing to the prevalence of lead and zinc-based pigments in historical works of art and their reactivity towards fatty acids in oil paints. Other types of fatty acid salts have also been documented in oil paints, including aluminum, cadmium, calcium, copper, magnesium, and sodium. Of these, calcium soaps have recently been detected during analyses at the Canadian Conservation Institute $(\mathrm{CCI})$ in both paintings and architectural paints [7], and have been responsible for significant conditions issues. Copper carboxylates are also well-documented, not only in the form of green copper resinate glazes (copper salts of resin acids), but also as soaps in oil paints applied to copper substrates [8].

The detailed characterization of metal soaps and their distribution within paint layers is of paramount importance if conservation intervention is to be successful. Fourier transform infrared (FTIR) spectroscopy is one of the leading analytical techniques for the study of metal soaps. Well-crystallized metal carboxylate salts give sharp, characteristic peaks in the mid-IR between ca. $1500-1600 \mathrm{~cm}^{-1}$ depending on the specific metal cation and fatty acid $[2,9]$. Ionomeric-type soaps, such as those often encountered in paint layers, give rise to broad absorption bands with obscured features that can make their definitive identification challenging. Further complicating their analysis and identification is the presence of more than one type of metal soap (different fatty acids and/or metal cation), which leads to further broadening and spectral overlap. Analytical methods with high spectral and spatial resolution are thus required to overcome these challenges and to properly characterize materials and objects containing metal soaps.

Cross-sectional samples of paint films offer a convenient platform on which analysis can be performed to identify the materials present in the layers of paint and how they relate to each other spatially. Infrared spectroscopy and infrared chemical mapping directly on painting cross-sections are valuable techniques for analyzing the distribution of pigments, fillers, binders and degradation products in paint layers. The analysis of cross-sections in transmission/absorption, attenuated total internal reflection (ATR), and external reflection configurations have all been reported. The analysis and chemical mapping of thin sections in transmission/absorption configuration [10-16] can generate very good results; however, it is not always possible to prepare thin sections suitable for transmission/absorption experiments from cultural heritage objects with varying porosity, friability, heterogeneity and relative hardness of the components [17]. The mapping of paint components in thick, resin-embedded cross-sections can be performed with ATR-FTIR [18-22], which involves simpler sample preparation than required for transmission/absorption and can also provide higher spatial resolutions. Contact of the internal reflecting element with the sample during ATR-FTIR measurements can sometimes cause deformation and a certain degree of damage to the surface of the sample, as well as the possibility of sample displacement and a loss of registration with visible images; however, these challenges are gradually being overcome and ATR-FTIR mapping is increasingly being used to investigate cultural heritage materials.

External reflection FTIR (R-FTIR) on thick, resinembedded cross-sections, in which contact with the sample is completely avoided, can also be used for the chemical mapping of paint components. R-FTIR mapping has been used to plot the distribution of paint components in cross-sections from a polychrome sculpture [23], murals [24, 25], easel paintings [26-29], and a painting on glass [30]. In most of these articles, mapping has been limited to the binding medium, pigments, and certain fillers. The identification and mapping of metal soaps in paintings by R-FTIR has not been extensively studied; only a few reports were found in the literature on the analysis and mapping of lead soaps [27, 28]. Much less has been reported on the analysis of other metal soaps by R-FTIR, including zinc, calcium, and copper soaps. One major challenge in the R-FTIR analysis of paint layers is that the reflected signal is usually much weaker than those obtained in transmission/absorption or ATRFTIR, and is very dependent on factors such as surface roughness. Reflection spectra can give rise to spectral distortions that can hinder spectral interpretation and comparisons to standard transmission/absorption reference libraries. While spectral correction algorithms are available to generate transmission/absorption-like spectra from reflection data, there is no consensus in the literature as to the validity of their application, especially in cases where both specular and diffuse components are present in the reflection signal, which would typically prevent the use of such corrections. 
This article describes the materials analysis of several cross-sections from paintings and painted objects from works in Canadian collections. Recent results on the use of R-FTIR spectroscopy to identify and map the distribution of paint components and especially metal soaps in cross-sections, including zinc, lead, copper, and calcium soaps, are presented. The results shown here are among the first detailed examinations of these metal soaps in paint cross-sections using R-FTIR spectroscopy. Additional surface polishing of prepared cross-sections was performed in order to maximize the specular reflectance from the samples, and the use of Kramers-Kronig spectral transformations (KKT) for the correction of reflection spectra is highlighted, and the results compared to those obtained in transmission/absorption on unmounted fragments of the same samples. In several cases, additional analysis using ATR-FTIR mapping is also shown.

\section{Experimental}

Infrared spectroscopy and chemical mapping were performed on samples from the following works: Equations in Space (1936) by Lawren Harris, L'Annonciation (1776) by Jean-Antoine Aide-Créquy, Le martyre de SaintPierre de Vérone (mid-nineteenth century) by Théophile Hamel, and a ceremonial copper object (T'lakwa) from the Kwakwaka'wakw First Nation. Microscopic paint samples were removed from each work and prepared as cross-sections by mounting a fragment of each sample in polyester resin followed by grinding and polishing using standard petrographic techniques. A final wet polishing step using an aqueous slurry of $0.05 \mu \mathrm{m}$ alumina particles was performed for each sample. The cross-sections were observed by incident light and fluorescence microscopy using a Leica DMRX microscope.

Gold-palladium reference mirrors for R-FTIR spectroscopy background measurements were deposited onto the surface of the polished polyester resin blocks using a Cressington 108 auto 7002-8 Sputter Coater. The crosssections were masked with aluminum foil held in place with adhesive tape to prevent metal deposition onto the samples, and a single $60 \mathrm{~s}$ deposition cycle was performed with a sputtering current of $40 \mathrm{~mA}$.

External reflection FTIR (R-FTIR) spectroscopy, undertaken at the Canadian Light Source synchrotron facility, was performed on the mounted cross-sections using an Agilent Cary 670 FTIR spectrometer and Cary 620 microscope system with a $25 \times$ objective $(\mathrm{NA}=0.82)$ and a $128 \times 128$ pixel liquid nitrogen cooled focal plane array (FPA) detector. This configuration allows a field of view of approximately $422.4 \mu \mathrm{m} \times 422.4 \mu \mathrm{m}(3.3 \mu \mathrm{m}$ pixel resolution/pixel area on the sample plane) and the simultaneous acquisition of 16,384 spectra. All spectra were collected between 3850 and $900 \mathrm{~cm}^{-1}$ with the coaddition of 512 scans at $4 \mathrm{~cm}^{-1}$ resolution. Background spectra were collected on the gold-palladium reference mirrors once before every sample. Chemical mapping was performed using Agilent Resolutions Pro 5.4 software. Single point spectra were compiled for each sample, and the false-colour intensity distributions of select compounds were generated according to the integrated absorbance of their characteristic spectral band(s). For all samples, Kramers-Kronig (KKT) corrections were applied to the R-FTIR spectra using Resolutions Pro. A colour scale from high value (white, red) to low value (blue) is employed.

For certain samples, ATR-FTIR spectroscopy and mapping was also performed on the mounted cross-sections using the Agilent Cary system with a Ge ATR crystal and a $64 \times 64$ pixel liquid nitrogen cooled FPA detector. This configuration allows a field of view of approximately $70.4 \mu \mathrm{m} \times 70.4 \mu \mathrm{m}(1.1 \mu \mathrm{m}$ pixel resolution/pixel area on the sample plane) and the simultaneous acquisition of 4096 spectra. All spectra were collected between 3850 and $900 \mathrm{~cm}^{-1}$ with the co-addition of 512 scans at $4 \mathrm{~cm}^{-1}$ resolution. Background spectra were collected in air once before every sample. The ATR crystal was brought into contact with the area of interest on the cross-section and the contact pressure was manually adjusted in order to optimize the signal intensity. Chemical mapping was performed using the same software as for R-FTIR.

Select paint layers were previously analyzed at the Canadian Conservation Institute (CCI) either directly on embedded cross-sections or as individual layers mechanically separated from unmounted paint fragments, using one or more of the following techniques: FTIR spectroscopy (in transmission/absorption mode), Raman spectroscopy, scanning electron microscopy/energy dispersive spectrometry (SEM/EDS), polarized light microscopy (PLM), and pyrolysis-gas chromatography-mass spectrometry (Py-GC-MS). The results of these previous analyses will only briefly be discussed here. For comparative FTIR spectra obtained in transmission/absorption mode, the samples were positioned on a diamond microsample cell and analyzed using a Bruker Hyperion 2000 microscope interfaced to a Tensor 27 spectrometer.

\section{Results \\ Equations in Space (1936) by Lawren Harris}

The oil on canvas painting Equations in Space by Group of Seven member Lawren Harris in the collection of the National Gallery of Canada (Accession number 15673) exhibits cracking, lifting and flaking of the paint, as well as disfiguring protrusions that have broken through the paint surface in some areas. A cross-section of a sample from the painting (Fig. 1) clearly shows the presence of 

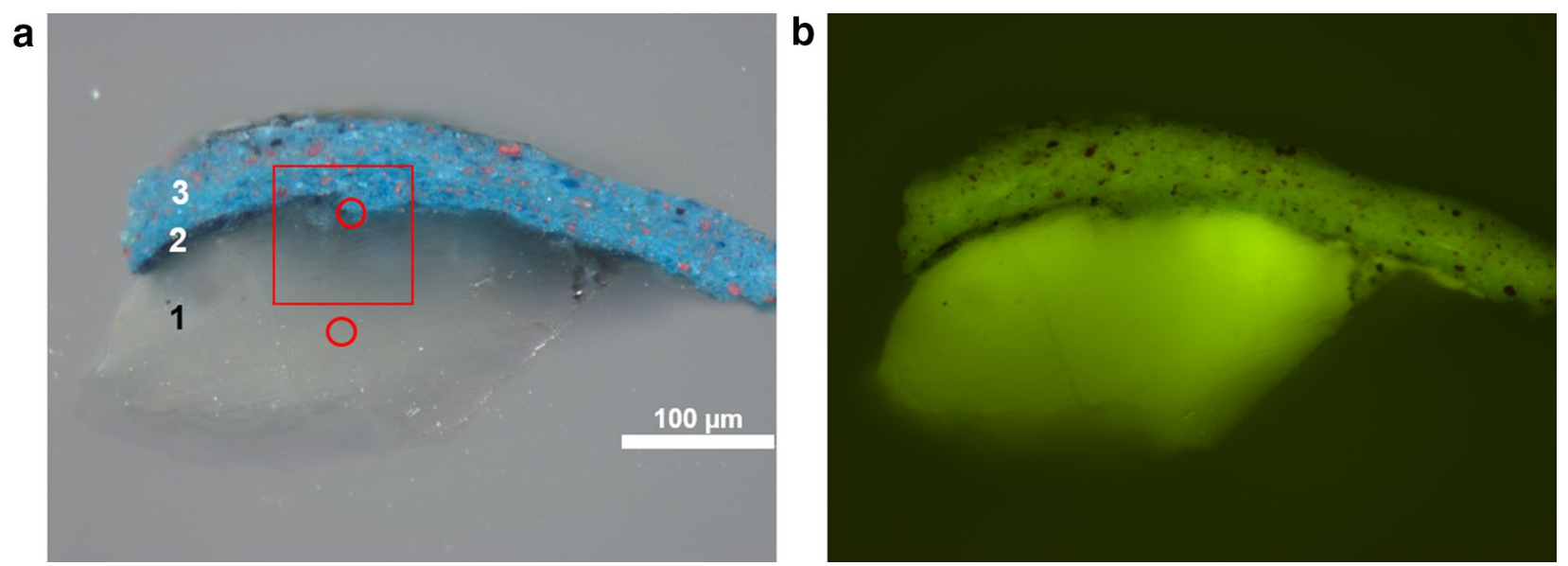

Fig. 1 Micrographs of the cross-section prepared from Equations in Space. a Incident light image. b Fluorescence image (blue excitation). The area analyzed by ATR-FTIR is shown within the red square in (a). Representative single-point spectra discussed in the text were taken from spots within the red circles

a translucent, waxy protrusion (1) beneath a thin blueblack layer (2) and an upper blue paint layer with red particles (3). Analysis of unmounted fragments of the sample determined that the protrusion (1) is composed primarily of zinc soaps with small quantities of free fatty acids and drying oil, and that the upper blue paint (3) contains lead white, Prussian blue, cobalt blue, and vermilion in a drying oil medium. Paint layers above the ground layers (not shown in the cross-section in Fig. 1) were found to contain a high concentration of zinc white and zinc soaps, and are undoubtedly the source of zinc in the protrusion [9].

A representative R-FTIR spectrum (KKT-corrected, $3200-900 \mathrm{~cm}^{-1}$ ) of a spot in the protrusion is shown in Fig. 2a. The spectrum shows a series of sharp peaks characteristic of zinc soaps, including a split carboxylate doublet at 1550 and $1533 \mathrm{~cm}^{-1}$, and a low intensity carbonyl peak at $1739 \mathrm{~cm}^{-1}$ attributed to glycerol esters of drying oil. The KKT-corrected R-FTIR spectrum is very similar to the transmission/absorption FTIR spectrum obtained on an unmounted fragment of the protrusion removed from the same sample (Fig. 2b). Similar carboxylate peak splitting has been reported in other works containing zinc soaps $[6,31]$. The peak splitting is due to a change in symmetry around the zinc cation compared to the bridging bidentate tetrahedral structure observed in synthesized zinc salts of saturated fatty acids. This could be caused by either a different coordination geometry or by a distortion of the tetrahedral structure around the zinc cation [9]. It has been hypothesized that the presence of mixed or hydrated soaps could prevent a compact packing configuration and lead to peak splitting [32]. Further work would be required to elucidate the mechanism of formation of the protrusion and the structure of zinc soaps showing this type of peak splitting.

The distribution of zinc soaps, obtained by integrating the zinc carboxylate bands between 1565 and $1516 \mathrm{~cm}^{-1}$, is shown in Fig. 2c. The results show that the soaps are localized entirely within the protrusion. The soaps are not present to any significant extent in the upper paint layers. The concentration of zinc soaps appears to be relatively uniform across the bulk of the protrusion, and then abruptly drops towards its edges.

External reflection FTIR (R-FTIR) spectra of the top blue paint layer (3) (not shown) showed the presence of lead white and Prussian blue. The distribution of lead white, obtained by integrating the broad carbonate band centered at $1441 \mathrm{~cm}^{-1}$, is shown in Fig. 2d. The image shows a high concentration of lead white in the upper blue paint layer (3), and a region of lower concentration between layer 3 and the zinc soap protrusion which may correspond to the thin blue-black layer (2). The distribution of Prussian blue, obtained by integrating its characteristic absorption peak at $2064 \mathrm{~cm}^{-1}$, is shown in Fig. 2e. The results show that the blue pigment is present in both layers 2 and 3.

ATR-FTIR spectroscopy and imaging were also performed on a smaller area of the cross-section to further examine the composition of the protrusion at the interface with the upper layers of paint. The area analyzed is shown within the red square in Fig. 1a. A representative ATR-FTIR spectrum of a point at the interface of the paint and the protrusion (Fig. 3a) shows the presence of Prussian blue $\left(2090 \mathrm{~cm}^{-1}\right)$, drying oil $\left(1735 \mathrm{~cm}^{-1}\right)$, zinc carboxylates (1548 and $\left.1529 \mathrm{~cm}^{-1}\right)$ and a broad carbonate band from lead white (centered at ca. $1398 \mathrm{~cm}^{-1}$ ). The 


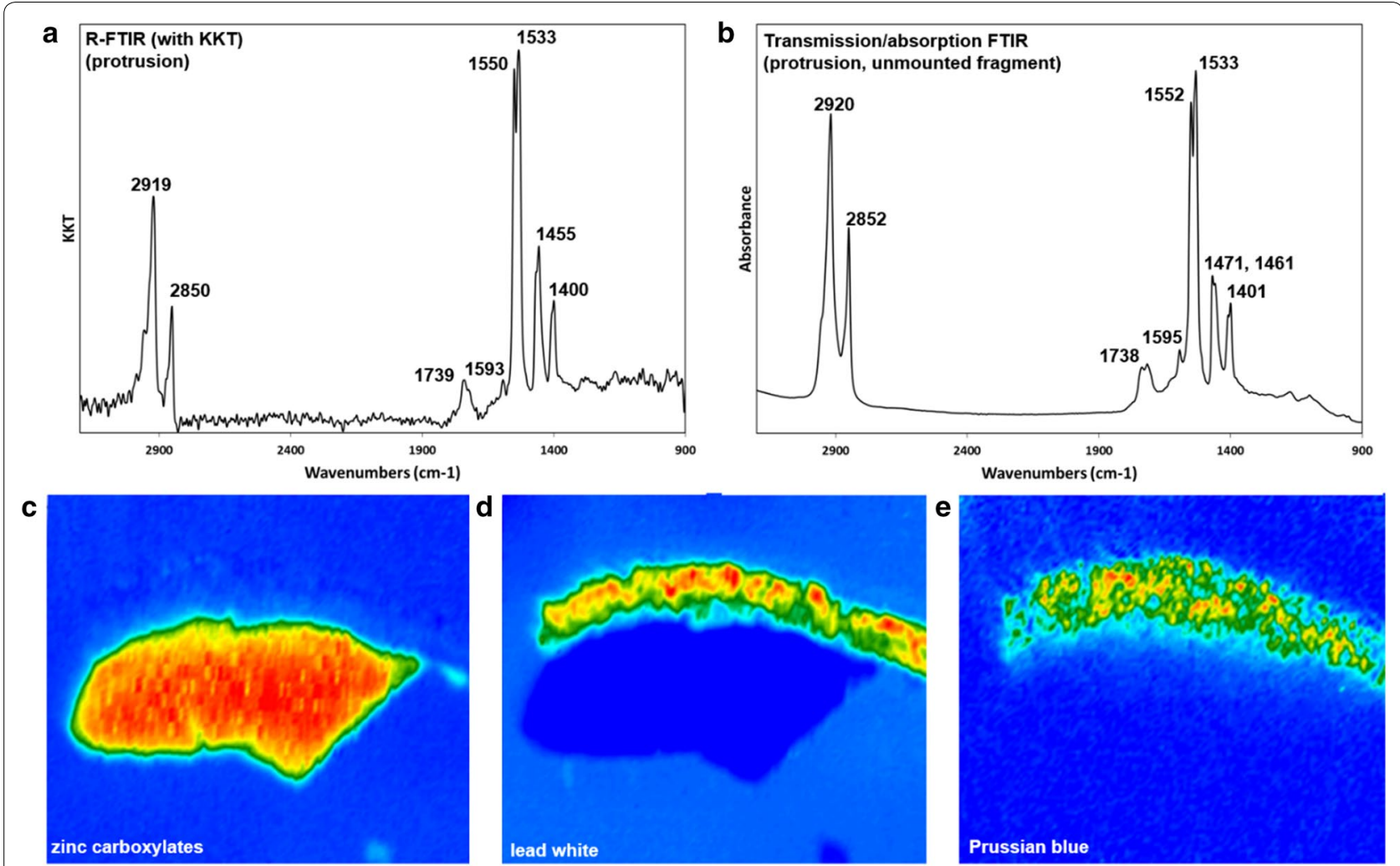

Fig. 2 Equations in Space. a Representative KKT-corrected R-FTIR spectrum of a spot in the protrusion (layer 1). b Transmission/absorption spectrum of an unmounted fragment of the protrusion. c Distribution of zinc soaps obtained by integrating the zinc carboxylate peaks in the R-FTIR spectrum $\left(1565-1516 \mathrm{~cm}^{-1}\right)$. d Distribution of lead white obtained by integrating the carbonate band in the R-FTIR spectrum $\left(1551-1351 \mathrm{~cm}^{-1}\right)$. e Distribution of Prussian blue obtained by integrating the cyano absorption peak in the R-FTIR spectrum (2112-2014 $\left.\mathrm{cm}^{-1}\right)$

distribution of lead white and Prussian blue, obtained by integrating their absorption peaks in the ATR-FTIR spectrum, is shown in Fig. 3b, c. The localization of these pigments within layers 2 and 3 is consistent with the results obtained by R-FTIR mapping, and clearly defines the boundary between the paint and the underlying protrusion. In a similar manner, the distribution of zinc soaps (Fig. 3d) also shows a defined interface between the protrusion and upper paint layers; the soaps are not present in significant concentrations in the layers of paint. On the other hand, integration of the carbonyl band at $1735 \mathrm{~cm}^{-1}$ associated with the glycerol esters in drying oil (as well as a shoulder at ca. $1705 \mathrm{~cm}^{-1}$ from carboxylic acid groups) (Fig. 3e) shows that the oil is not only present in the paint layers, but is also present in minor quantities within the outer edge of the protrusion. Certain areas in the protrusion at the interface with the upper paint layers show differences in the carboxylate band (Fig. 3f; spot 1 and spot 2 are shown in Fig. 3d); some areas have a single peak centered at $1535 \mathrm{~cm}^{-1}$, while others have the same split peak observed in the bulk of the protrusion. This suggests that there are differences in the symmetry around the zinc cation near the interface between the protrusion and the paint.

\section{L'Annonciation (1776) by Jean-Antoine Aide-Créquy}

The painting L'Annonciation by Jean-Antoine AideCréquy is an oil on canvas in the collection of the Fabrique de Notre-Dame de Bonsecours de l'Islet, in l'Islet, Quebec, Canada. While the painting was undergoing conservation treatment, cross-sections were taken for analysis to determine the materials and technique of the artist [33]. In one cross-section, taken from the dark green background of the painting, a large waxy protrusion was observed at the top of the sample. The crosssection (Fig. 4) shows a red ground layer (1), a dark green paint layer (2), and the waxy protrusion (3). Analysis of unmounted fragments of the sample indicates that the protrusion is composed primarily of lead soaps with small amounts of lead white and drying oil. The underlying paint layers were not fully characterized, but were found to contain lead (possibly from lead white or a leadbased drier), iron, calcium, silicon, and aluminum by SEM/EDS. It is possible that the lead-based component 
a

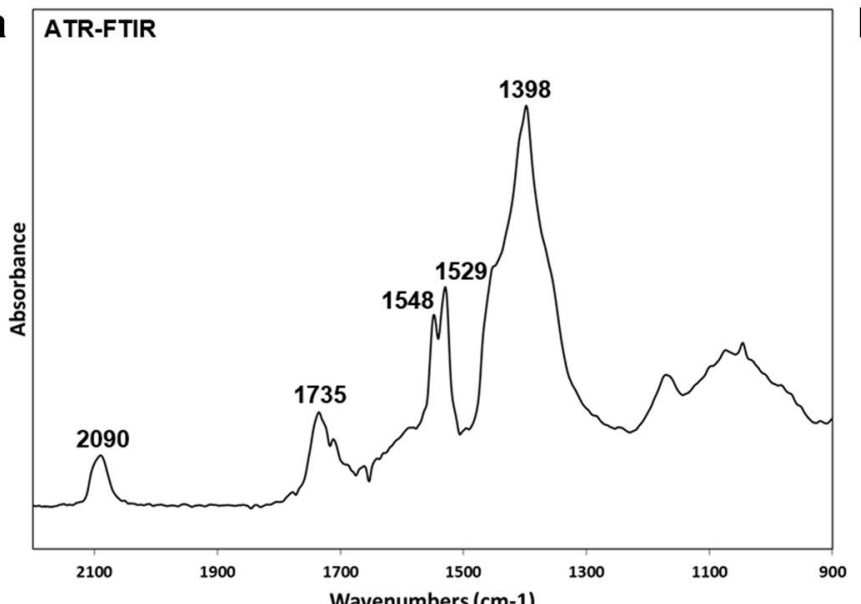

b

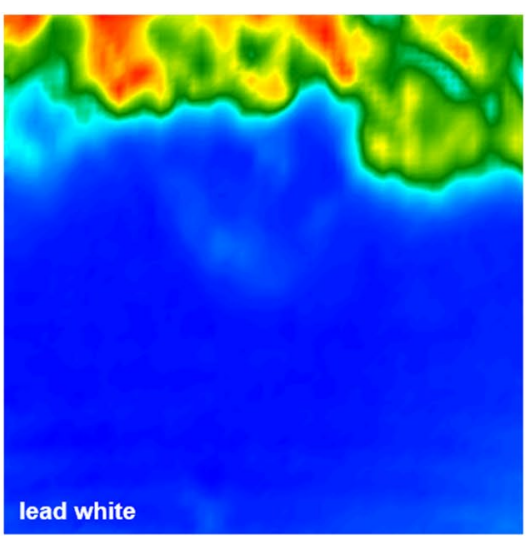

d

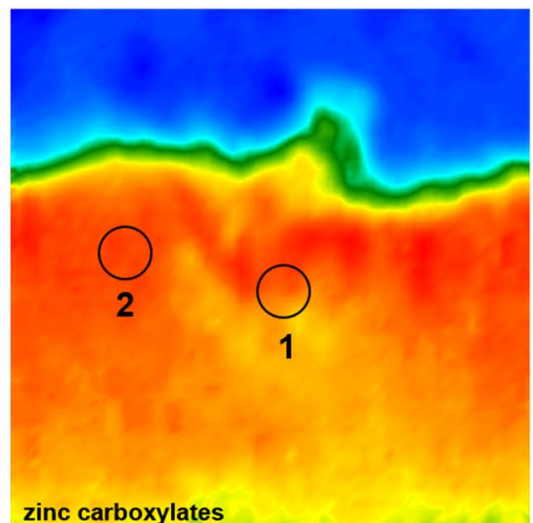

f

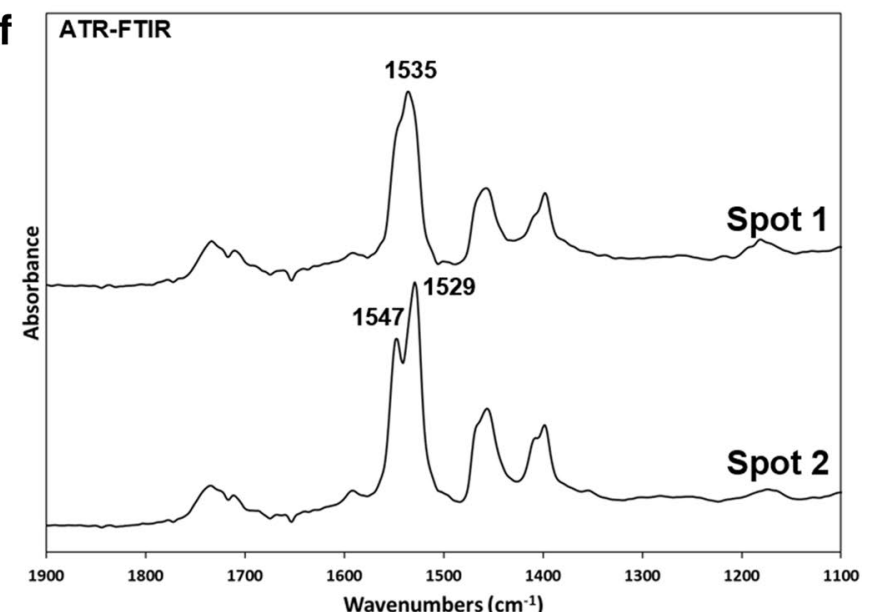

Fig. 3 Equations in Space. a Representative ATR-FTIR spectrum from a spot near the interface of the protrusion and the upper paint layers. $\mathbf{b}$ Distribution of lead white obtained by integrating the carbonate band in the ATR-FTIR spectrum $\left(1485-1271 \mathrm{~cm}^{-1}\right)$. c Distribution of Prussian blue obtained by integrating the cyano peak in the ATR-FTIR spectrum $\left(2130-2048 \mathrm{~cm}^{-1}\right)$. $\mathbf{d}$ Distribution of zinc soaps obtained by integrating the zinc carboxylate peaks in the ATR-FTIR spectrum $\left(1568-1505 \mathrm{~cm}^{-1}\right)$. e Distribution of drying oil obtained by integrating the carbonyl band in the ATR-FTIR spectrum $\left(1789-1676 \mathrm{~cm}^{-1}\right)$. The black line demarks the approximate interface between the protrusion and the paint layers. $\mathbf{f}$ Representative ATR-FTIR spectra of spots 1 and 2 shown in (d) 
in the dark green paint layer is the source of lead in the protrusion.

A representative R-FTIR spectrum (KKT-corrected, $3200-900 \mathrm{~cm}^{-1}$ ) of an area near the surface of the protrusion is shown in Fig. 5a. The spectrum shows a mixture of lead white with peaks at 1405 and $1045 \mathrm{~cm}^{-1}$ and a sharp peak at $1519 \mathrm{~cm}^{-1}$ characteristic of lead carboxylates. The KKT-corrected R-FTIR spectrum is in very good agreement with the transmission/absorption spectrum from an unmounted sample of the protrusion (Fig. 5b). A KKT-corrected R-FTIR spectrum from a spot further into the bulk of the protrusion (Fig. 5c) shows that the area is composed almost entirely of lead carboxylates, with a small carbonyl peak from drying oil as well. There are no bands associated with lead white in this area. The presence of areas composed almost entirely of lead soaps in the protrusion could not be ascertained from transmission/absorption spectra on samples removed from the protrusion, likely owing to the specific material sampled for analysis in transmission/absorption mode and the heterogeneity of the protrusion. This highlights the usefulness of FTIR spectroscopy directly on cross-sections to obtain compositional information on very small size scales or in layers with a heterogeneous composition.

The distribution of lead soaps, obtained by integrating the carboxylate band at $1519 \mathrm{~cm}^{-1}$, is shown in Fig. 6a. The results show a high concentration of soaps near the interface of the protrusion with the underlying paint layers, as well as smaller quantities extending towards the surface of the protrusion. The soaps do not appear to extend all the way to the surface of the protrusion, but gradually decrease in concentration as the distance from the paint/protrusion interface increases. As well, the lead soaps do not extend significantly below the protrusion into the dark green paint layer or into the red ground. The presence of the lead soaps at the interface with the underlying paint layer is consistent with the lead-based component in the dark green layer being the source of the soaps. Integration of the broad carbonate band at $1405 \mathrm{~cm}^{-1}$ shows the distribution of lead white (Fig. 6c); it is present in small amounts in the underlying paint layers, and in much higher concentrations in the protrusion, especially near the surface. The presence of high concentrations of lead white at the surface of the protrusion could be attributed to mineralization processes inside the lead soap aggregate, with atmospheric $\mathrm{CO}_{2}$ being the carbonate source $[27,34]$.

A small area of the cross-section was also analyzed by ATR-FTIR to further examine the composition at the interface of the protrusion with the paint layers. The area analyzed is shown within the red square in Fig. 4a. ATR-FTIR spectra from different areas within the whitish protrusion (not shown) were similar to those obtained in reflectance. The distribution of lead soaps and lead white obtained from the integration of their respective absorption bands in the ATR-FTIR spectra are shown in Fig. 6b, d, and show similar results as above. The lead soaps are present in highest concentration within the protrusion near the interface with the underlying paint layers (shown as the narrow redcolored band in Fig. 6b). The chemical mapping with ATR-FTIR also clearly shows that the lead soaps do not extend into the lower brown paint layer, and that their concentration abruptly drops at the interface between the protrusion and the dark green layer.

\section{Le martyre de Saint-Pierre de Vérone (mid-nineteenth century) by Théophile Hamel}

The painting Le martyre de Saint-Pierre de Vérone is an oil on canvas in the collection of the Musée national des beaux-arts du Québec (Accession number 2013.21). The painting was undergoing conservation treatment aimed at addressing significant paint delamination and losses. In some of the losses, a translucent brown/beige material was present under the ground layers. A cross-section from an area with significant paint losses (Fig. 7) shows this brownish translucent layer (1) beneath two white ground layers (2 and 3), a blue paint layer (4), a gray layer (5) and a thin dark layer on the surface, likely dirt (6). Analysis of unmounted paint fragments showed that the bottom brownish layer is composed primarily of calcium soaps, and that the ground layers are composed of calcium carbonate and lead white [7].

A representative KKT-corrected R-FTIR spectrum $\left(1900-1100 \mathrm{~cm}^{-1}\right)$ of a spot in the lower translucent brown layer (1) is shown in Fig. 8a. The spectrum shows a series of sharp peaks characteristic of calcium soaps, including the carboxylate peaks at 1578 and $1542 \mathrm{~cm}^{-1}$. Again, the KKT-corrected R-FTIR spectrum is very similar to the transmission/absorption FTIR spectrum obtained on a sample of the brown material removed from within a loss on the painting (Fig. 8b). KKTcorrected R-FTIR spectra of areas in the two white ground layers (not shown) were consistent with results obtained in transmission/absorption, dominated by a very broad carbonate band from calcium carbonate and lead white. In certain areas of the second ground layer, however, the R-FTIR spectrum shows a distinct peak at $1518 \mathrm{~cm}^{-1}$ characteristic of lead soaps (Fig. 8c). Lead soaps were not identified in the FTIR transmission/ absorption spectrum of an unmounted fragment of this layer (Fig. 8d), in which only dominant carbonate species were detected. This again highlights the ability of FTIR spectroscopy on cross-sections to identify 

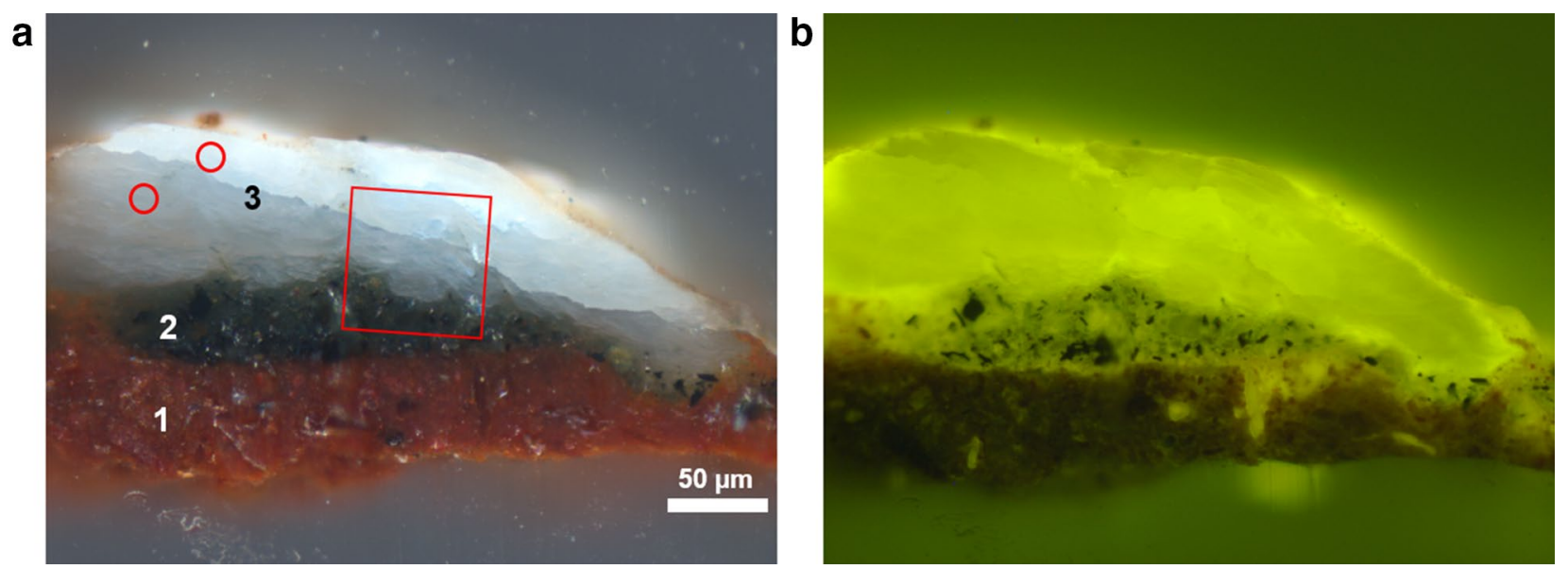

Fig. 4 Micrographs of the cross-section prepared from L'Annonciation. a Incident light image. b Fluorescence image (blue excitation). The area analyzed by ATR-FTIR is shown within the red square in (a). Representative single-point spectra discussed in the text were taken from spots within the red circles
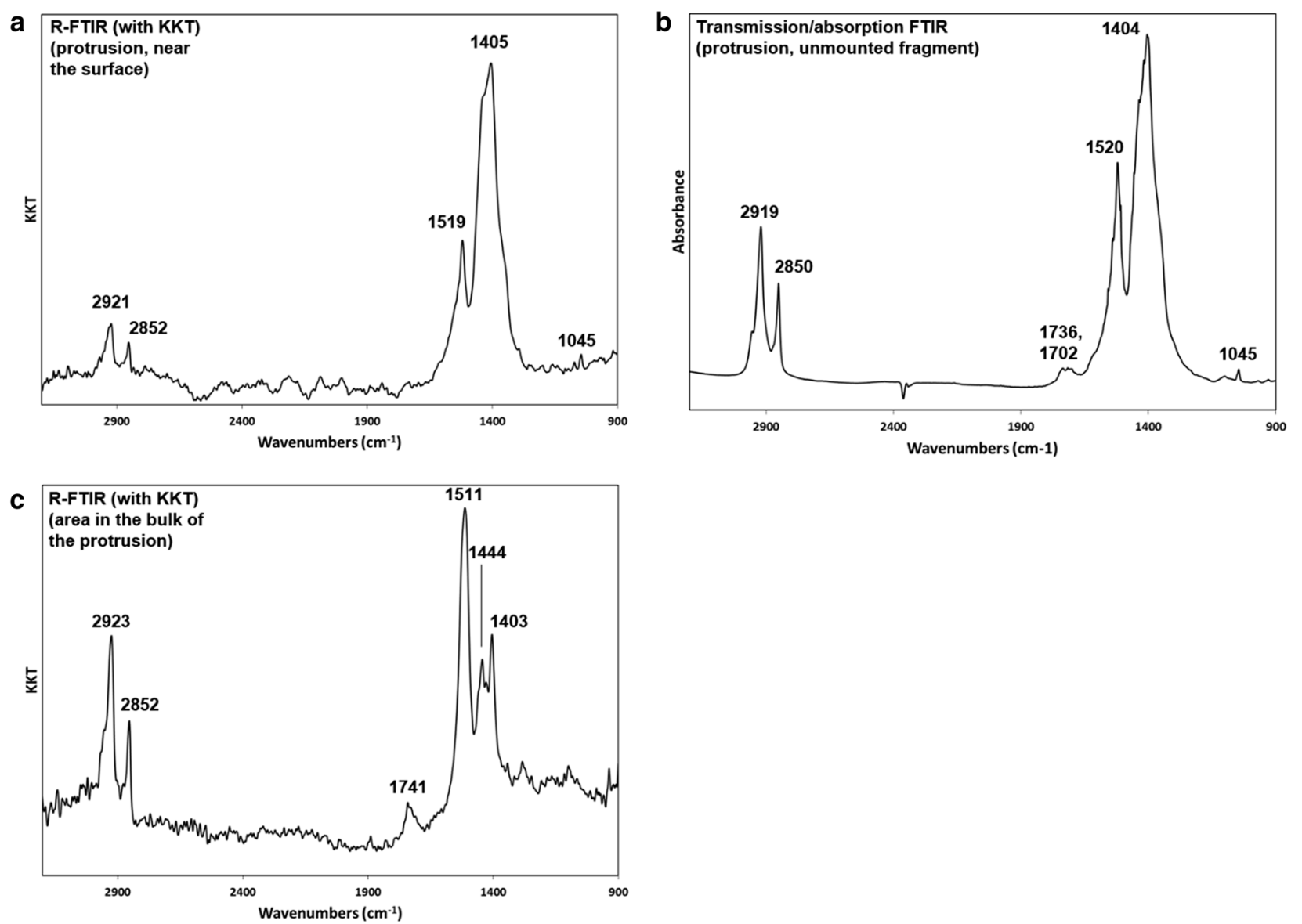

Fig. 5 L'Annonciation. a Representative KKT-corrected R-FTIR spectrum of a spot near the surface of the protrusion (layer 3). b Transmission/ absorption spectrum of an unmounted fragment of the protrusion. c KKT-corrected R-FTIR spectrum of a spot in the bulk of the waxy protrusion (layer 3) 
a

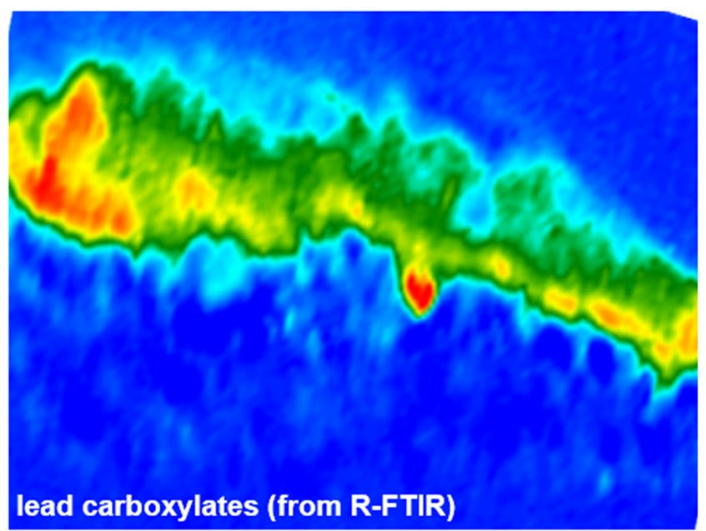

C

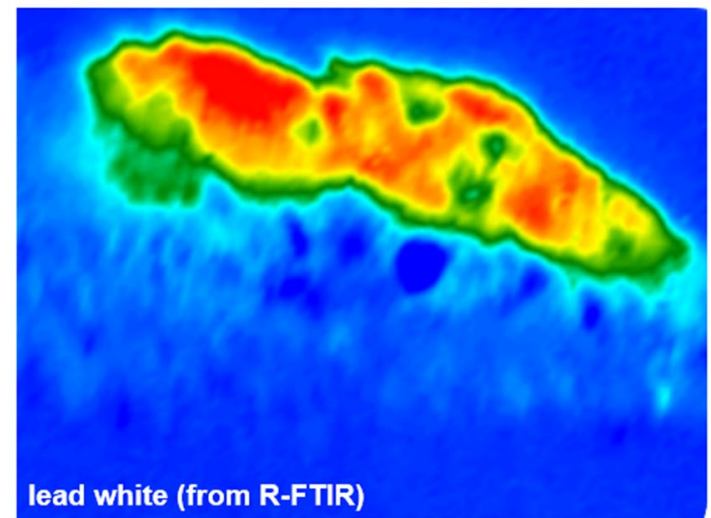

b

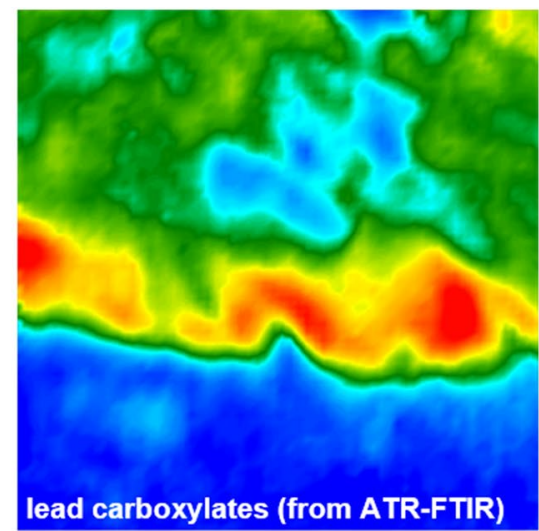

d

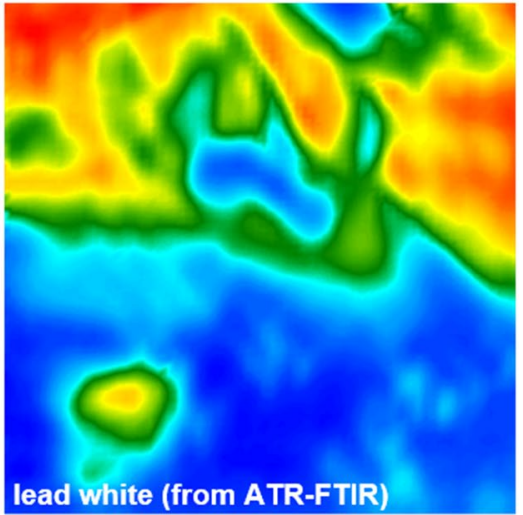

Fig. 6 L'Annonciation. a Distribution of lead soaps obtained by integrating the lead carboxylate peak in the R-FTIR spectrum $\left(1576-1480 \mathrm{~cm}^{-1}\right)$. b Distribution of lead soaps obtained by integrating the lead carboxylate peak in the ATR-FTIR spectrum $\left(1557-1475 \mathrm{~cm}^{-1}\right)$. c Distribution of lead white obtained by integrating the carbonate band in the R-FTIR spectrum $\left(1484-1325 \mathrm{~cm}^{-1}\right)$. $\mathbf{d}$ Distribution of lead white obtained by integrating the carbonate band in the ATR-FTIR spectrum $\left(1472-1265 \mathrm{~cm}^{-1}\right)$

localized species in heterogeneous layers, even those present in low concentrations.

The distribution of calcium soaps, obtained by integrating the calcium carboxylate band at $1578 \mathrm{~cm}^{-1}$, is shown in Fig. 9a. Only the higher energy calcium carboxylate peak was used for integration in order to minimize spectral overlap with bands from lead carboxylates. The results illustrate that the calcium soaps are localized entirely within layer 1 in the right hand portion of the layer. Integration of the lead carboxylate band centered at $1518 \mathrm{~cm}^{-1}$ shows the presence of lead soaps throughout layer 3 (Fig. 9b), and possibly in minor quantities in the grey paint layer (5) and at the interface between layers 1 and 2. Interestingly, Fig. 9b shows that the lead soaps are almost completely absent from the first ground layer (2) as well as the blue paint layer (4), even though both of these layers were found to contain major quantities of lead by SEM/EDS elemental analysis and previous analysis by FTIR spectroscopy confirmed that layer 2 contains drying oil and lead white.

\section{Ceremonial copper object (T'lakwa)}

\section{from the Kwakwaka'wakw First Nation}

Prior to conservation treatment, a painted Kwakwaka'wakw First Nations ceremonial copper object (T'lakwa) from the Pacific Northwest in Canada exhibited significant cracking and lifting of the black paint, which had flaked off in several areas. The object was in a fire while on exhibit at the U'mista Cultural Centre in Alert Bay, British Columbia. Although it was not directly burned or damaged by water from the fire suppression system, it was exposed to high heat, soot, and steam for several hours. Analysis of unmounted fragments of the black paint identified a mixture of traditional materials, including fat, oil, resin, charcoal, and calcium carbonate, as well as appreciable amounts of fatty acid salts, including calcium and copper soaps. A fragment of the delaminated paint was prepared as a cross-section (Fig. 10), which shows a distinct thick white/beige layer between two layers of black paint. 

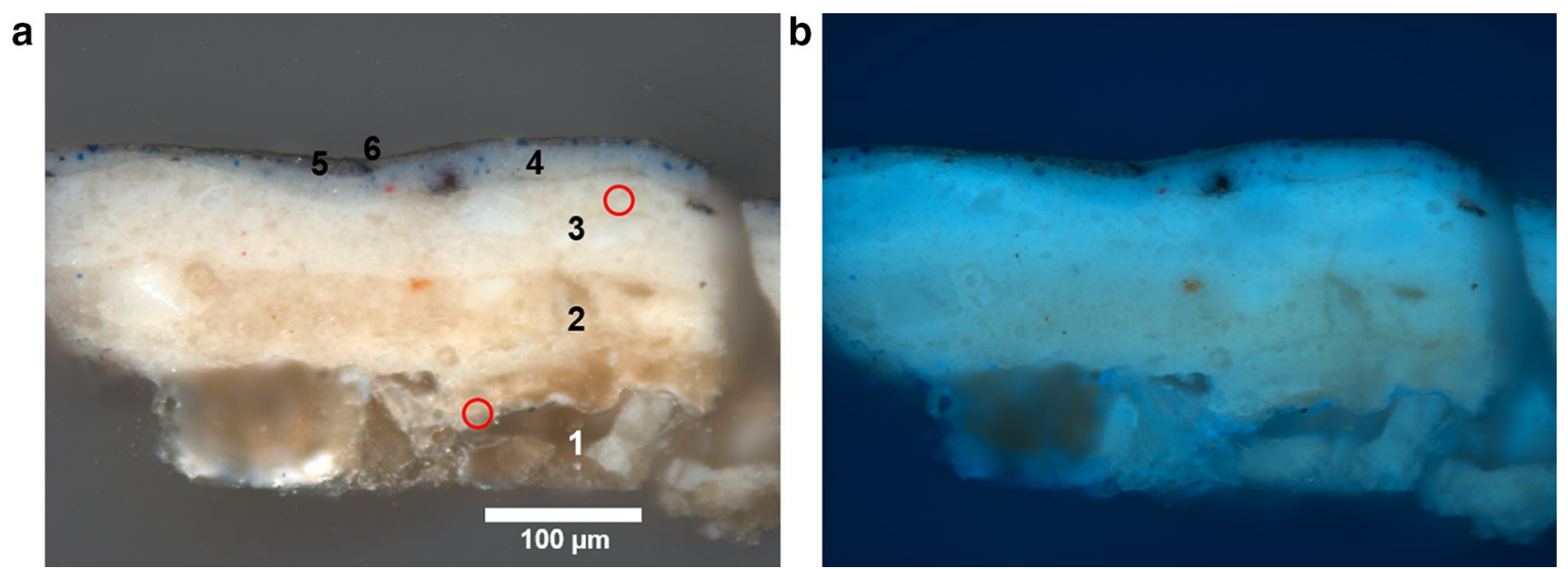

Fig. 7 Micrographs of the cross-section prepared from Le martyre de Saint-Pierre de Vérone. a Incident light image. b Fluorescence image (UV excitation). Representative single-point spectra discussed in the text were taken from spots within the red circles
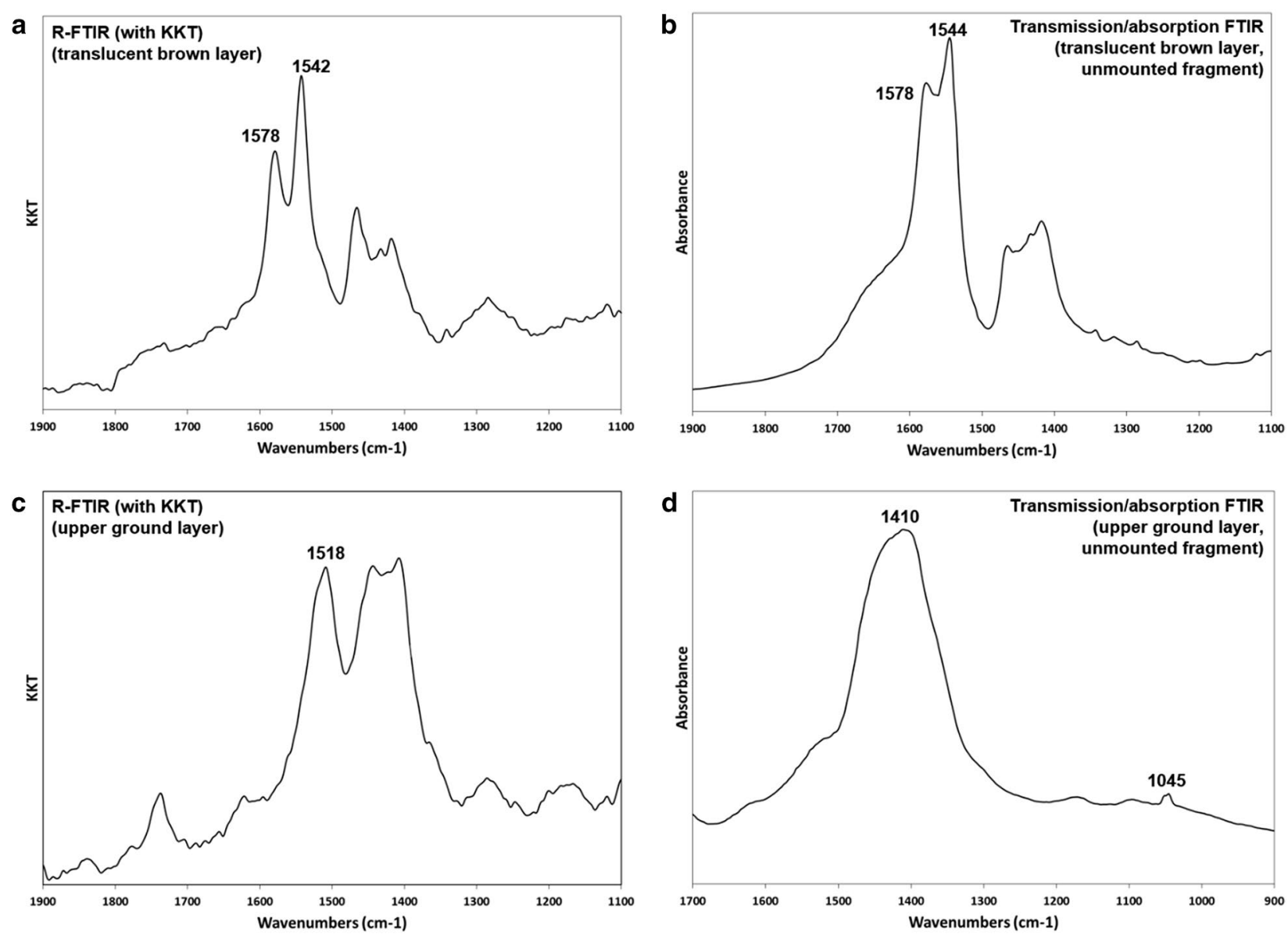

Fig. 8 Le martyre de Saint-Pierre de Vérone. a Representative KKT-corrected R-FTIR spectrum of a spot in the bottom translucent brown layer (layer 1). b Transmission/absorption spectrum of an unmounted fragment of the translucent brown layer. c Representative KKT-corrected R-FTIR spectrum of a spot in the upper ground layer (layer 3). $\mathbf{d}$ Transmission/absorption spectrum of an unmounted fragment of the upper ground layer 
a

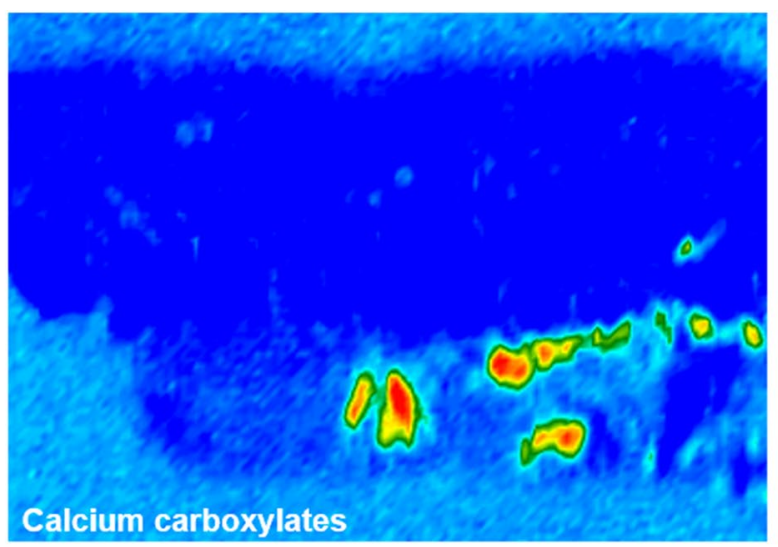

b

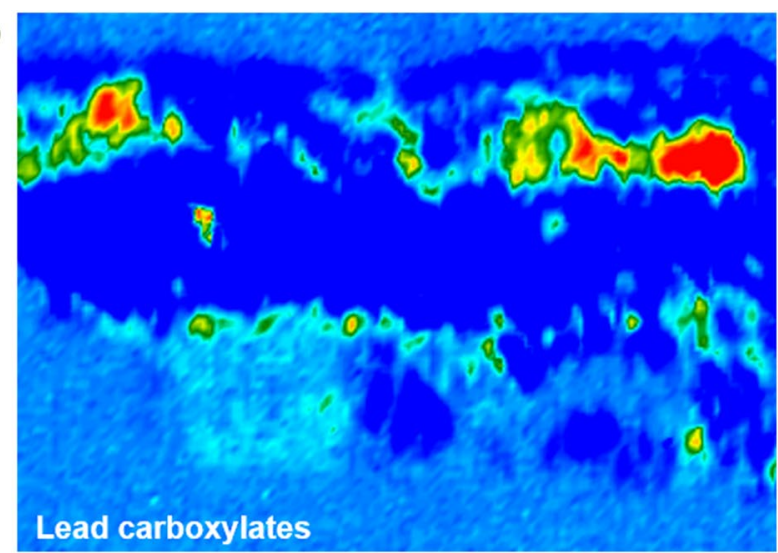

Fig. 9 Le martyre de Saint-Pierre de Vérone. a Distribution of calcium soaps obtained by integrating the high energy calcium carboxylate peak in the R-FTIR spectrum (1612-1560 $\mathrm{cm}^{-1}$ ). b Distribution of lead soaps obtained by integrating the lead carboxylate peak in the R-FTIR spectrum $\left(1561-1485 \mathrm{~cm}^{-1}\right)$
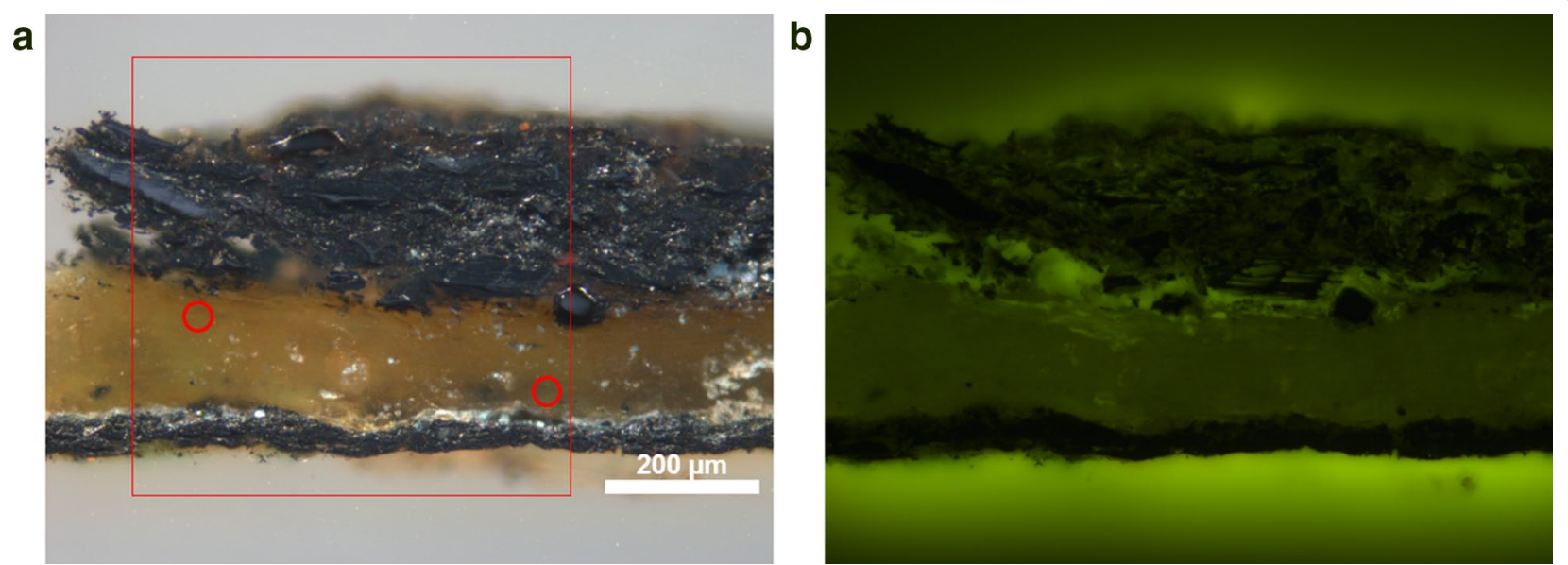

Fig. 10 Micrographs of the cross-section prepared from the T'lakwa painted copper. a Incident light image. b Fluorescence image (blue excitation). The area analyzed by R-FTIR is shown within the red square in (a). Representative single-point spectra discussed in the text were taken from spots within the red circles

The cross-section was analyzed by R-FTIR spectroscopy; the area analyzed is shown within the red square in Fig. 10a. Representative KKT-corrected R-FTIR spectra $\left(3200-900 \mathrm{~cm}^{-1}\right)$ of different spots in the beige layer are shown in Fig. 11a, b. The reflectance spectrum of an area analyzed in the upper portion of the beige layer (Fig. 11a) shows peaks corresponding to calcium soaps, including the carboxylate peaks at 1576 and $1541 \mathrm{~cm}^{-1}$. The spectrum of an area in the lower portion of the beige layer (Fig. 11b) shows sharp peaks corresponding to copper soaps, including the carboxylate peaks at 1593 and $1544 \mathrm{~cm}^{-1}$. The carboxylate region of the spectra is shown in greater detail in Fig. 11c. The KKT-corrected R-FTIR spectra are similar to transmission/absorption
FTIR spectra obtained on different fragments of the beige material, in which both calcium and copper carboxylates were identified (Fig. 11d).

The distribution of the two soap species, obtained by the integration of their respective carboxylate bands, is shown in Fig. 12a (calcium) and b (copper). In order to determine the spatial distribution of these different metal soaps, narrow spectral windows were used for the integration to avoid spectral overlap. The images show that the beige layer is composed of regions with high concentrations of calcium soaps and other regions with high concentrations of copper soaps. In fact, the distribution of the two species within the beige layer seems to be inversely related; in areas with high calcium soap 

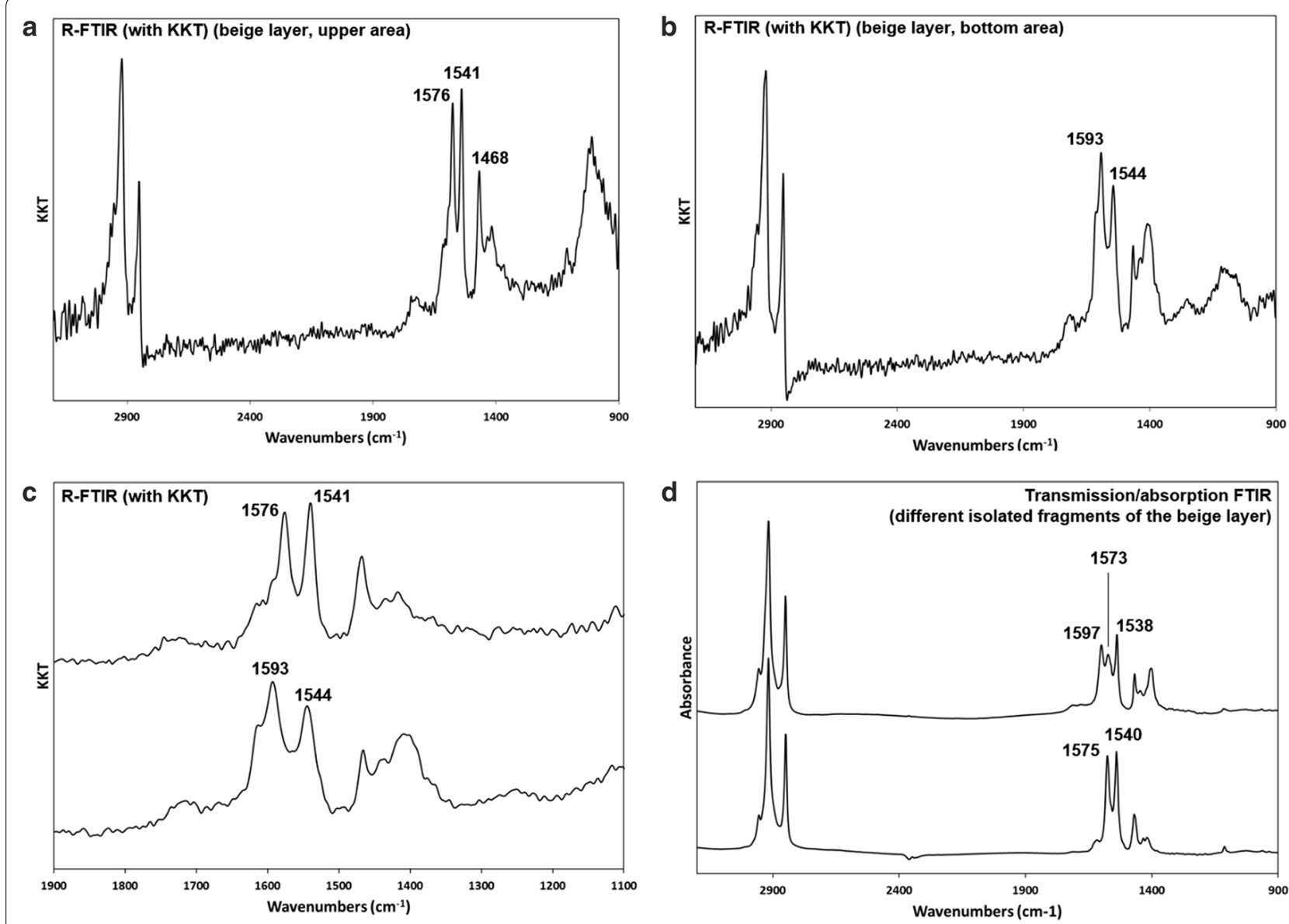

Fig. 11 T'lakwa painted copper. a Representative KKT-corrected R-FTIR spectrum of a spot in the upper portion of the beige layer. b Representative KKT-corrected R-FTIR spectrum of a spot in the lower portion of the beige layer. $\mathbf{c}$ Details of the metal carboxylate region of the spectra shown in (a, b). $\mathbf{d}$ Transmission/absorption spectra of different unmounted fragments of the beige layer

\section{a}

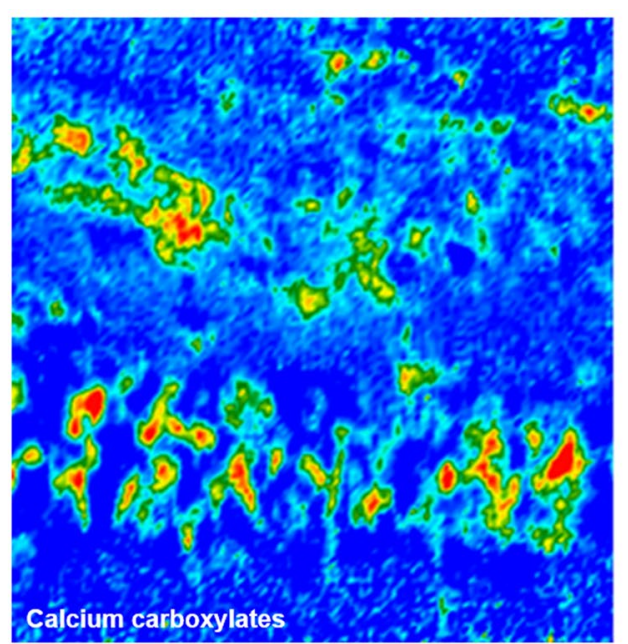

b

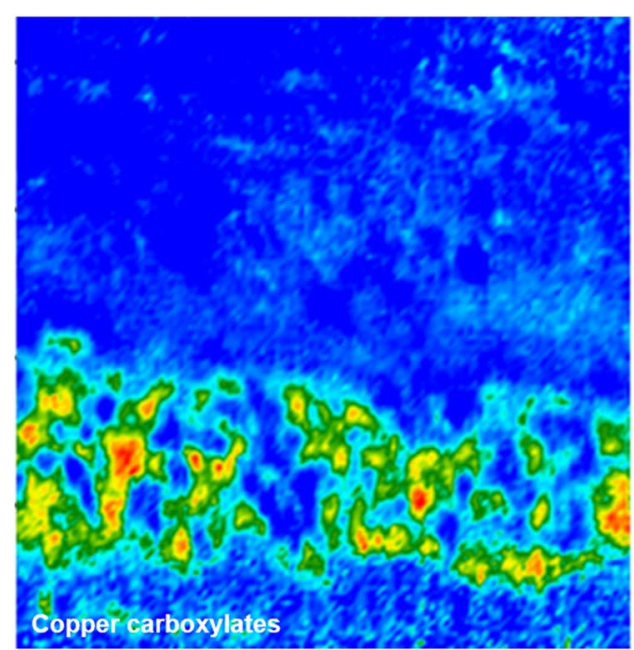

Fig. 12 T'lakwa painted copper. a Distribution of calcium soaps obtained by integrating the calcium carboxylate peak in the R-FTIR spectrum $\left(1585-1563 \mathrm{~cm}^{-1}\right)$. b Distribution of copper soaps obtained by integrating the copper carboxylate peak in the R-FTIR spectrum $\left(1625-1585 \mathrm{~cm}^{-1}\right)$ 
concentration, the copper soap concentration is very low; in areas with high copper soap concentration, the calcium soap concentration is very low. This suggests that the metal soaps form small clusters within the beige layer, as opposed to a homogenous mixture. In addition, the images show that while calcium soaps are also located in the upper black paint layer, the copper soaps are present only in the beige layer and the bottom black paint layer, likely owing to their closer proximity to the copper substrate.

\section{Discussion}

The case studies presented here show that R-FTIR and ATR-FTIR spectroscopy directly on cross-sectional paint samples allowed for the identification of several pigments, the oil binding medium, and especially metal soap degradation products. Zinc, lead, calcium, and copper fatty acid salts were all readily identified in the examples shown. While previous articles have also shown the identification and mapping of drying oil and pigments by R-FTIR spectroscopy [23-30], the results shown here are among the first detailed examinations of these metal soaps in paint cross-sections using R-FTIR. The use of a focal plane array (FPA) detector provided resolution close to the diffraction limit, and allowed for the mapping of these species across entire cross-sections. In several cases, the high spatial resolution allowed for the identification of species that were not detected in single point transmission/absorption spectra of unmounted fragments of the same samples, highlighting the usefulness of FTIR spectroscopy directly on cross-sections to obtain compositional information on these very small size scales, even of species present in low concentration.

A significant challenge in external reflection FTIR spectroscopy is the generation of both specular and diffuse reflection from sample surfaces and the resulting spectral distortions that accompany these phenomena. In these cases, spectral corrections cannot be applied, and the reflection spectra are quite challenging to interpret and cannot readily be compared to standard transmission/absorption reference databases. Several articles have reported chemometric methods including principal component analysis to map paint components using uncorrected reflectance spectra [23, 24, 35]. However, as shown here, the use of highly polished samples in which specular reflection is dominant can allow for KKT spectral transformations to be applied to generate transmission/absorption-like spectra, which significantly facilitate the identification of the components in the paint layers. In fact, in most of the examples shown here, the KKTcorrected R-FTIR spectra were almost indistinguishable from transmission/absorption spectra. Not all paint samples may be suitable for the substantial amount of polishing required to produce the smooth surfaces that give rise to predominantly specular reflection. However, as shown here, this method seems very well suited for mapping of metal soaps.

In the examples where ATR-FTIR was also performed, the spectra were very similar to those obtained in external reflectance. Although the specific instrumental configuration used for this study was restricted to a much smaller field of view, the ATR mapping results were very useful, especially to provide compositional details at the interface of paint layers and degradation products, due to the slight increase in magnification and improved spatial resolution created by using the Ge ATR element. In particular, in the cross-section prepared from the painting Equations in Space, results from ATR-FTIR mapping show differences in the zinc carboxylate band near the interface between the paint and the protrusion. For certain samples, shifting and tilting of the sample-embedding resin blocks upon contact with the ATR crystal prevented the acquisition of reliable spectra. Nevertheless, with improved sample preparation techniques these challenges can surely be overcome.

\section{Conclusion}

External reflection FTIR spectroscopy was performed on several cross-sections from paintings and painted objects from works in Canadian collections to identify and map the distribution of paint components and metal soap degradation products. Zinc, lead, calcium, and copper fatty acid salts were all readily identified, along with numerous pigments and the oil binding medium. The results shown here are among the first detailed examinations of these metal soaps in paint cross-sections by R-FTIR spectroscopy. The use of highly polished samples in which specular reflection is dominant allowed for KKT spectral transformations to be applied to generate transmission/ absorption-like spectra which facilitated identification of these species. ATR-FTIR spectroscopy was also performed on several samples, which provided additional compositional details at the interface of paint layers and degradation products.

\section{Abbreviations}

FTIR: Fourier transform infrared spectroscopy; R-FTIR: external reflectance Fourier transform infrared spectroscopy; ATR-FTIR: attenuated total internal reflectance Fourier transform infrared spectroscopy; KKT: Kramers-Kronig transformations; CCl: Canadian Conservation Institute; SEM/EDS: scanning electron microscopy/energy dispersive $x$-ray spectrometry; PLM: polarized light microscopy; Py-GC-MS: pyrolysis-gas chromatography-mass spectrometry; FPA: focal plane array.

\section{Acknowledgements}

The authors would like to acknowledge the conservators involved in the treatment of these works and the institutions to which the works belong for providing the samples used in this study. Jennifer Poulin, Marie-Claude Corbeil, 
Elizabeth Moffatt, and Dominique Duguay are thanked for contributing to the previous analyses of samples from these works.

\section{Authors' contributions}

All authors read and approved the final manuscript.

\section{Funding}

Funding for the work performed at the Canadian Conservation Institute was provided by the Government of Canada, Department of Canadian Heritage. Some of the research described in this paper was performed at the Mid-Infrared Spectromicroscopy (01B1-1) Beamline at the Canadian Light Source, which is supported by the Canada Foundation for Innovation, Natural Sciences and Engineering Research Council of Canada, the University of Saskatchewan, the Government of Saskatchewan, Western Economic Diversification Canada, the National Research Council Canada, and the Canadian Institutes of Health Research.

\section{Availability of data and materials}

The datasets used and/or analysed during the current study are available from the corresponding author on reasonable request.

\section{Competing interests}

The authors declare that they have no competing interests.

\section{Author details \\ ${ }^{1}$ Canadian Conservation Institute, 1030 Innes Rd., Ottawa, ON K1B 4S7, 2V3, Canada \\ Received: 25 June 2019 Accepted: 3 September 2019 Published online: 13 September 2019} Canada. ${ }^{2}$ Canadian Light Source Inc., 44 Innovation Blvd., Saskatoon, SK S7N

\section{References}

1. Noble P. A brief history of metal soaps in paintings from a conservation perspective. In: Casadio F, et al., editors. Metals soaps in art. Cham: Springer; 2019. p. 1-22.

2. Robinet $\mathrm{L}$, Corbeil M-C. The characterization of metal soaps. Stud Conserv. 2003;48(1):23-40.

3. Hermans JJ, Keune K, Van Loon A, ledema PD. Toward a complete molecular model for the formation of metal soaps in oil paints. In: Casadio F, et al., editors. Metals soaps in art. Cham: Springer; 2019. p. 47-67.

4. van den Berg KJ, Burnstock A, Schilling M. Notes on metal soap extenders in modern oil paints: history, use, degradation, and analysis. In: Casadio F, et al., editors. Metals soaps in art. Cham: Springer; 2019. p. 329-42.

5. Cotte M, Checroun E, De Nolf W, Taniguchi Y, De Viguerie L, Burghammer M, Walter P, Rivard C, Salomé M, Janssens K, Susini J. Lead soaps in paintings: friends or foes. Stud Conserv. 2017;62(1):2-23.

6. Osmond G. Zinc soaps: an overview of zinc oxide reactivity and consequences of soap formation in oil-based paintings. In: Casadio F, et al., editors. Metals soaps in art. Cham: Springer; 2019. p. 25-46.

7. Helwig K, Forest É, Turcotte A, Baker W, Binnie NE, Moffatt E, Poulin J. The formation of calcium fatty acid salts in oil paint: two case studies. In: Casadio F, et al., editors. Metals soaps in art. Cham: Springer; 2019. p. 297-311.

8. Pavlopoulou L-C, Watkinson D. The degradation of oil painted copper surfaces. Stud Conserv. 2006;51(sup1):55-65.

9. Helwig K, Poulin J, Corbeil M-C, Moffatt E, Duguay D. Conservation issues in several twentieth-century canadian oil paintings: the role of zinc carboxylate reaction products. In: van den Berg KJ, et al., editors. Issues in contemporary oil paint. Cham: Springer; 2014. p. 167-84.

10. Pouyet E, Cotte M, Fayard B, Salomé M, Meirer F, Mehta A, Uffelman ES, Hull A, Vanmeert F, Kieffer J, Burghammer M, Janssens K, Sette F, Mass J. 2D X-ray and FTIR micro-analysis of the degradation of cadmium yellow pigment in paintings of Henri Matisse. Appl Phys A. 2015;121:967-80.

11. Papliaka ZE, Vaccari L, Zanini F, Sotiropoulou S. Improving FTIR imaging speciation or organic compound residues or their degradation products in wall painting samples, by introducing a new thin section preparation strategy based on cyclododecane pre-treatment. Anal Bioanal Chem. 2015:407:5393-403.
12. Cotte M, Checroun E, Susini J, Walter P. Micro-analytical study of interactions between oil and lead compounds in paintings. Appl Phys A. 2007:89:841-8

13. Echard J-P, Cotte M, Dooryhee E, Bertrand L. Insights into the varnishes of historical musical instruments using synchrotron micro-analytical methods. Appl Phys A. 2008;92:77-81.

14. Lluveras A, Boularand S, Andreotti A, Vendrell-Saz M. Degradation of azurite in mural paintings: distribution of copper carbonate, chlorides and oxalates by SRFTIR. Appl Phys A. 2010;99:363-75.

15. Mass J, Sedlmair J, Patterson CS, Carson D, Buckley B, Hirschmugl C. SR-FTIR imaging of the altered cadmium sulfide yellow paints in Henri Matisse's Le Bonheur de Vivre (1905-6) —examination of visually distinct degradation regions. Analyst. 2013;138:6032-43.

16. Osmond G, Boon JJ, Puskar L, Drennan J. Metal stearate distributions in modern artists' oil paints: surface and cross-sectional investigation of reference paint films using conventional and synchrotron infrared microspectroscopy. Appl Spectrosc. 2012;66(10):1136-44.

17. Unger M, Mattson E, Patterson CS, Alavi Z, Carson D, Hirschmugl CJ. Synchrotron-based multiple-beam FTIR chemical imaging of a multi-layered polymer in transmission and reflection: towards cultural heritage applications. Appl Phys A. 2013;111(1):135-45.

18. Spring M, Ricci C, Peggie DA, Kazarian SG. ATR-FTIR imaging for the analysis of organic materials in paint cross sections: case studies on paint samples from the National Gallery, London. Anal Bioanal Chem. 2008:392:37-45.

19. Joseph E, Ricci C, Kazarian SG, Mazzeo R, Prati S, loele M. Macro-ATRFT-IR spectroscopic imaging analysis of paint cross-sections. Vib Spectrosc. 2010;53:274-8.

20. Joseph E, Prati S, Sciutto G, loele M, Santopadre P, Mazzeo R. Performance evaluation of mapping and linear imaging FTIR microspectroscopy for the characterisation of paint cross sections. Anal Bioanal Chem. 2010;396:899-910.

21. Kaszowska Z, Malek K, Panczyk M, Mikolajska A. A joint application of ATR-FTIR and SEM imaging with high spatial resolution: identification and distribution of painting materials and their degradation products in paint cross sections. Vib Spectrosc. 2013;65:1-11.

22. Gabrieli F, Rosi F, Vichi A, Cartechini L, Buemi LP, Kazarian SG, Miliani C. Revealing the nature and distribution of metal carboxylates in Jackson Pollock's Alchemy (1947) by micro-attenuated total reflection FT-IR spectroscopic imaging. Anal Chem. 2017;89:1283-9.

23. Rosi F, Federici A, Brunetti BG, Sgamellotti A, Clementi S, Miliani C. Multivariate chemical mapping of pigments and binders in easel painting cross-sections by micro IR reflection spectroscopy. Anal Bioanal Chem. 2011;399:3133-45.

24. Prati S, Rosi F, Sciutto G, Oliveri P, Catelli E, Miliani C, Mazzeo R. Evalualtion of the effect of different paint cross section preparation methods on the performances of fourier transform infrared microscopy in total reflection mode. Microchem J. 2013;110:314-9.

25. Nevin A, Melia JL, Osticioli I, Gautier G, Colombini MP. The identification of copper oxalates in a 16th century Cypriot exterior wall painting using micro FTIR, micro Raman spectroscopy and gas chromatography-mass spectrometry. J Cult Heritage. 2008;9:154-61.

26. van Loon A, Boon JJ. Characterization of the deterioration of bone black in the 17th century Oranjezaal paintings using electron-microscopic and micro-spectroscopic imaging techniques. Spectrochim Acta Part B. 2004;59:1601-9.

27. Keune K, Boon JJ. Analytical imaging studies of cross-sections of paintings affected by lead soap aggregate formation. Stud Conserv. 2007;52:161-76.

28. van der Weerd J, Brammer H, Boon JJ, Heeren RMA. Fourier transform infrared microscopic imaging of an embedded paint cross-section. Appl Spectrosc. 2002;56(3):275-83.

29. Poli T, Chiantore O, Giovagnoli A, Piccirillo A. FTIR imaging investigation in MIR and in an enlarged MIR-NIR spectral range. Anal Bioanal Chem. 2012;402:2977-84.

30. Staniszewska E, Malek K, Kaszowska Z. Studies on paint cross sections of a glass painting by using FT-IR and Raman microspectroscopy supported by univariate and hierarchical cluster analyses. J Raman Spectrosc. 2013;44:1144-55. 
31. Hermans JJ, Keune K, van Loon A, ledema PD. An infrared spectroscopic study of the nature of zinc carboxylates in oil paintings. J Anal At Spectrom. 2015;30:1600-8.

32. Osmond G. Zinc white and the influence of paint composition for stability in oil based media. In: van den Berg KJ, et al., editors. Issues in contemporary oil paint. Cham: Springer; 2014. p. 263-81.

33. Forest É, Corbeil M-C, Moffatt E. Jean-Antoine Aide-Créquy (1749-1780): matériaux et technique picturale. J Can Art Hist. 2015;36(2):11-48.

34. Keune K. Binding medium, pigments and metal soaps characterised and localised in paint cross-sections. Doctoral Thesis submitted to the FOM Institute for Atomic and Molecular Physics, Amsterdam. 2015:163-4.
35. Capobianco G, Bracciale MP, Sali D, Sbardella F, Belloni P, Bonifazi G, Serranti S, Santarelli ML, Guidi MC. Chemometrics approach to FT-IR hyperspectral imaging analysis of degradation products in artwork crosssection. Microchem J. 2017;132:69-76.

\section{Publisher's Note}

Springer Nature remains neutral with regard to jurisdictional claims in published maps and institutional affiliations.

\section{Submit your manuscript to a SpringerOpen ${ }^{\circ}$ journal and benefit from:}

- Convenient online submission

- Rigorous peer review

- Open access: articles freely available online

- High visibility within the field

- Retaining the copyright to your article

Submit your next manuscript at $\boldsymbol{\nabla}$ springeropen.com 